\title{
CHAOS IN A NON-AUTONOMOUS INDUCTORLESS ACTIVE-RC CIRCUIT
}

\author{
MUHAMMAD TAHER ABUELMA'ATTI \\ and MAZEN M. JOHAR \\ King Fahd University of Petroleum and Minerals \\ Box 203 Dhahran 31261 Saudi Arabia
}

\begin{abstract}
Chaotic performance of a simple inductorless active-RC non-autonomous electronic circuit is reported. The circuit uses two operational amplifiers, an operational transconductance amplifier, a diode, two resistors, and one capacitor only. No discrete inductors are involved.
\end{abstract}

\section{INTRODUCTION}

At present, there is a growing interest in studying non-autonomous electronic circuits exhibiting chaotic behavior [1-6]. A circuit is said to be non-autonomous if it is driven by an ac source. The circuit reported by Matsumoto et al. [1] is the simplest non-autonomous circuit that can become chaotic. It is formed of a linear resistor, a linear inductor and a non-linear capacitor. It appears, therefore, that use of an inductor is inevitable to obtain chaotic behavior in a non-linear electronic circuit.

On the other hand, inductance simulations using operational-amplifier-based active- $R C$ realizations are very attractive for monolithic realization. This raises the question of whether it is feasible to obtain chaotic behavior from active-RC realizations using operational amplifiers and/or operational transconductance amplifiers, capacitors, and resistors only.

It is the objective of this paper to report experimental results showing that chaotic behavior can occur in an inductorless active-RC circuit.

\section{PROPOSED CIRCUIT}

The proposed circuit is shown in Fig. 1. A breadboard of the circuit was constructed using off-the-shelf components. The phase trajectory of $V_{B}$ versus $V_{A}$ was measured with an oscilloscope in the X-Y mode. The results, obtained with the component values shown in Fig. 1, are shown in Fig. 2. The circuit of Fig. 1 was derived by a 3300A Hewlett-Packard function generator with an output impedance of $600 \Omega$. Fig. 2(a) shows the phase trajectory obtained with a square-wave input, Fig. 2(b) shows the phase trajectory obtained with a sinusoidal input, and Fig. 2(c) shows the phase trajectory obtained with a triangular input.

These observations confirm that the proposed inductorless active-RC circuit of Fig. 1 can provide chaotic performance by careful adjustment of the values of the resistances. It must be mentioned here that the two dc sources are simultaneously 


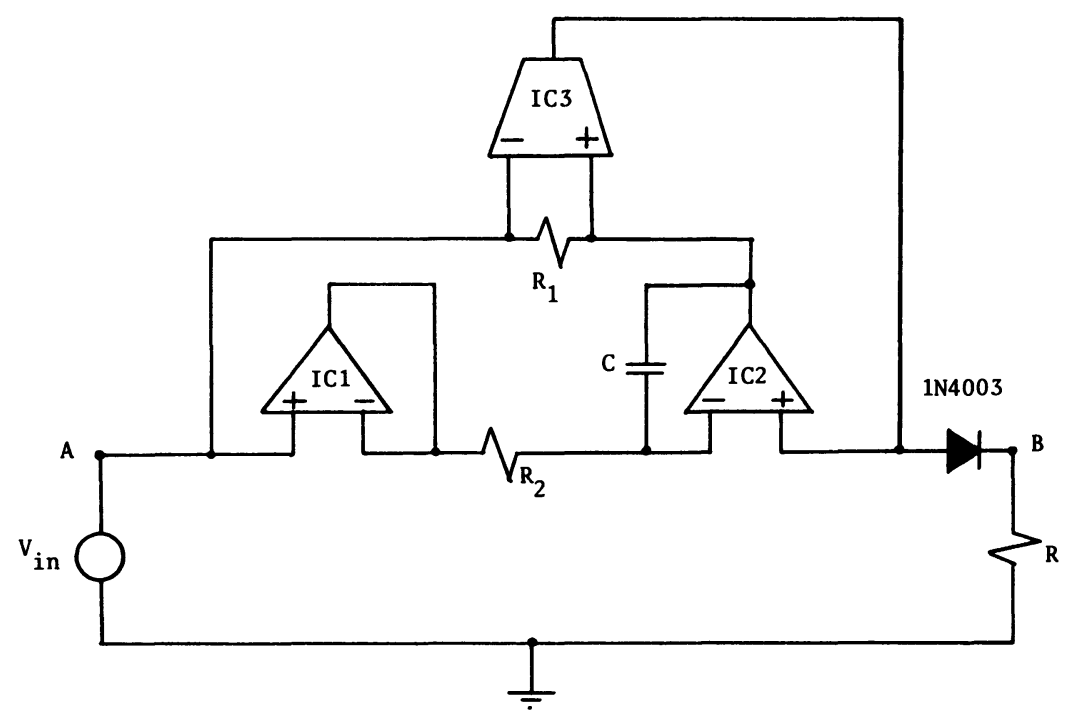

FIGURE 1 Proposed inductorless nonautonomous chaotic circuit $R_{1}=2 K, R_{2}=5 K, R=15 \Omega$, C $=1 \mathrm{nF}$ IC1 and IC2: 741 OA, IC3: 3080 OTA

used to supply the two operational amplifiers, as well as the operational transconductance amplifier.

In an attempt to explore the similarity between the proposed circuit of Fig. 1 and the Matsumoto et al. circuit [1], let the operational amplifiers and the operational transconductance amplifier be ideal. Routine analysis, assuming that $g_{m} R_{1}=1$, where $g_{m}$ is the transconductance of the operational transconductance amplifier, shows that the input impedance between points $X$ and $Y$ of the circuit shown in Fig. 3 can be expressed as [7]

$Z_{\text {inp }}=\frac{j \omega C R_{1} R_{2}}{1+j \omega C R_{2}}$

This is equivalent to the impedance of a parallel $R_{e q}-L_{e q}$ circuit with

$L_{e q}=C R_{1} R_{2}$

and

$R_{e q}=R_{1}$

Thus, the equivalent circuit of the proposed circuit of Fig. 1 can be represented by the circuit shown in Fig. 4. From Fig. 4 it appears that the similarity between the proposed circuit of Fig. 1 and Matsumoto et al. circuit [1] is very close.

\section{CONCLUSION}

In this paper, the experimental observations of the chaotic performance of an inductorless active-RC electronic circuit have been reported. This is probably the first attempt to obtain chaotic performance from a non-autonomous circuit without 
(a)

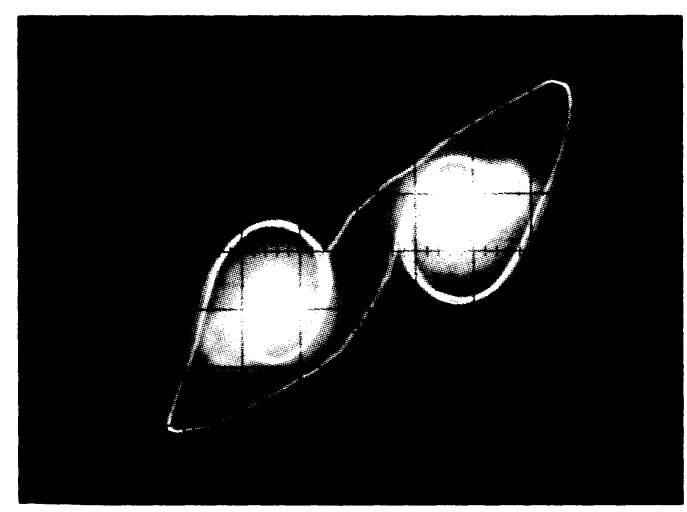

(b)

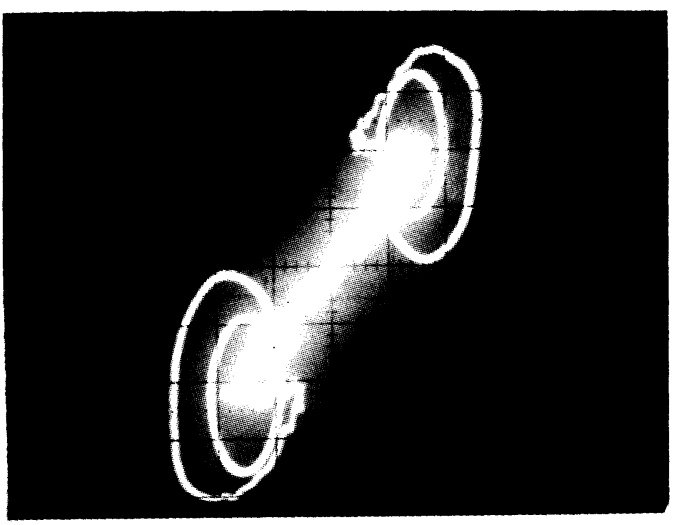

\section{(c)}

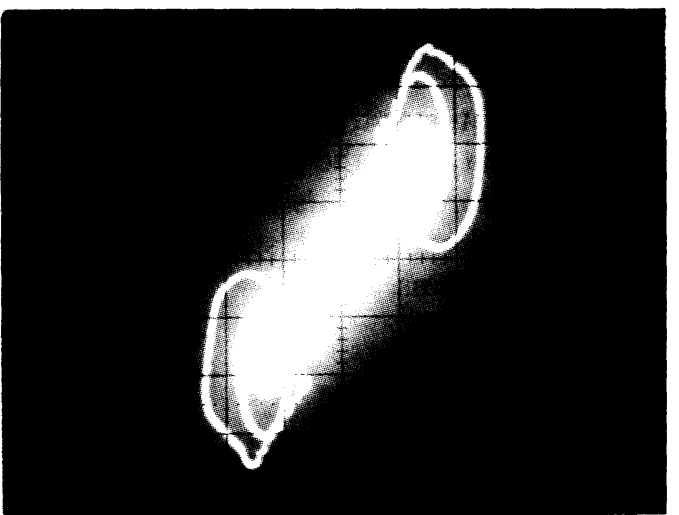

FIGURE 2 Phase trajectories with (a) square wave input (b) sinusoidal input and (c) triangular input 


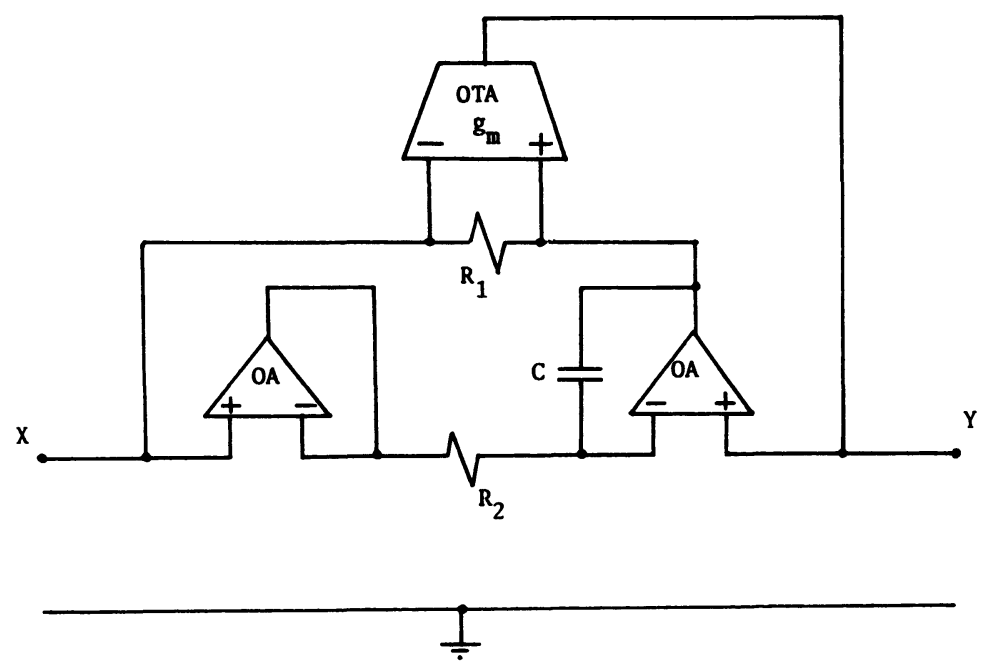

FIGURE 3 Circuit used for inductor simulation $g_{m} R_{1}=1$.

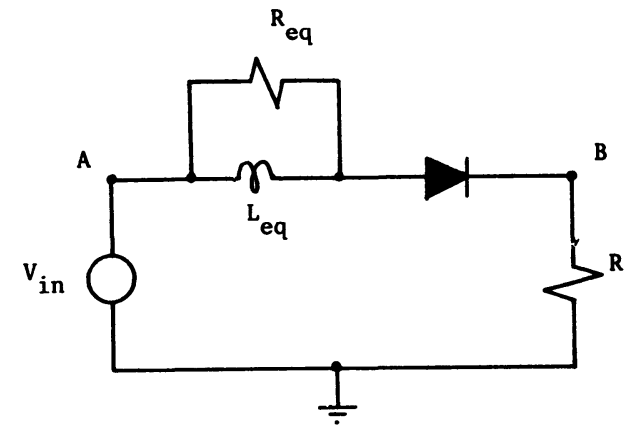

FIGURE 4 Equivalent circuit of the proposed circuit shown in Fig. 1.

using discrete inductors. The similarity between the proposed circuit of Fig. 1 and the Matsumoto et al. [1] circuit has been illustrated assuming ideal models for the operational amplifiers and the operational transconductance amplifier.

Finally, it is worth mentioning that since the proposed circuit of Fig. 1 does not use any externally connected inductors, then its implementation in an integrated circuit form is feasible. This may be a useful step towards the integration of chaos-generating circuits in VLSI systems.

\section{REFERENCES}

1. T. Matsumoto, L.O. Chua and S. Tanaka, Simplest chaotic nonautonomous circuit, Physical Review A, Vol. 30, 1984, pp. 1155-1157

2. A. Azzouz, R. Duhr and M. Hasler, IEEE Transactions on Circuits and Systems, Vol. CAS-31, 1984, pp. $587-588$

3. D.C. Hamill, Learning about chaotic circuits with SPICE, IEEE Transactions on Education, Vol. 36,1993 , pp. 28-35 
4. D.J. Jefferies, G.G. Johnstone and J.H.B. Deane, An experimental search for the conditions for the existence of chaotic states in class $\mathrm{C}$ bipolar transistor RF amplifiers, International Journal of Electronics, Vol. 71, 1991, pp. 661-673

5. K. Murali and M. Lakshmanan, Chaotic dynamics of the driven Chua's circuit, IEEE Transactions on Circuits and Systems-I: Fundamental Theory and Applications, Vol. 40, 1993, pp. 836-840

6. A. Azzouz, R. Duhr and M. Hasler, Transition to chaos in a simple nonlinear circuit driven by a sinusoidal voltage source, IEEE Transactions on Circuits and Systems, Vol. CAS-30, 1983, pp. 913-914

7. R. Senani, New single-capacitor simulations of floating inductors, Electrocomponent Science and Technology, Vol. 10, 1982, pp. 7-12 

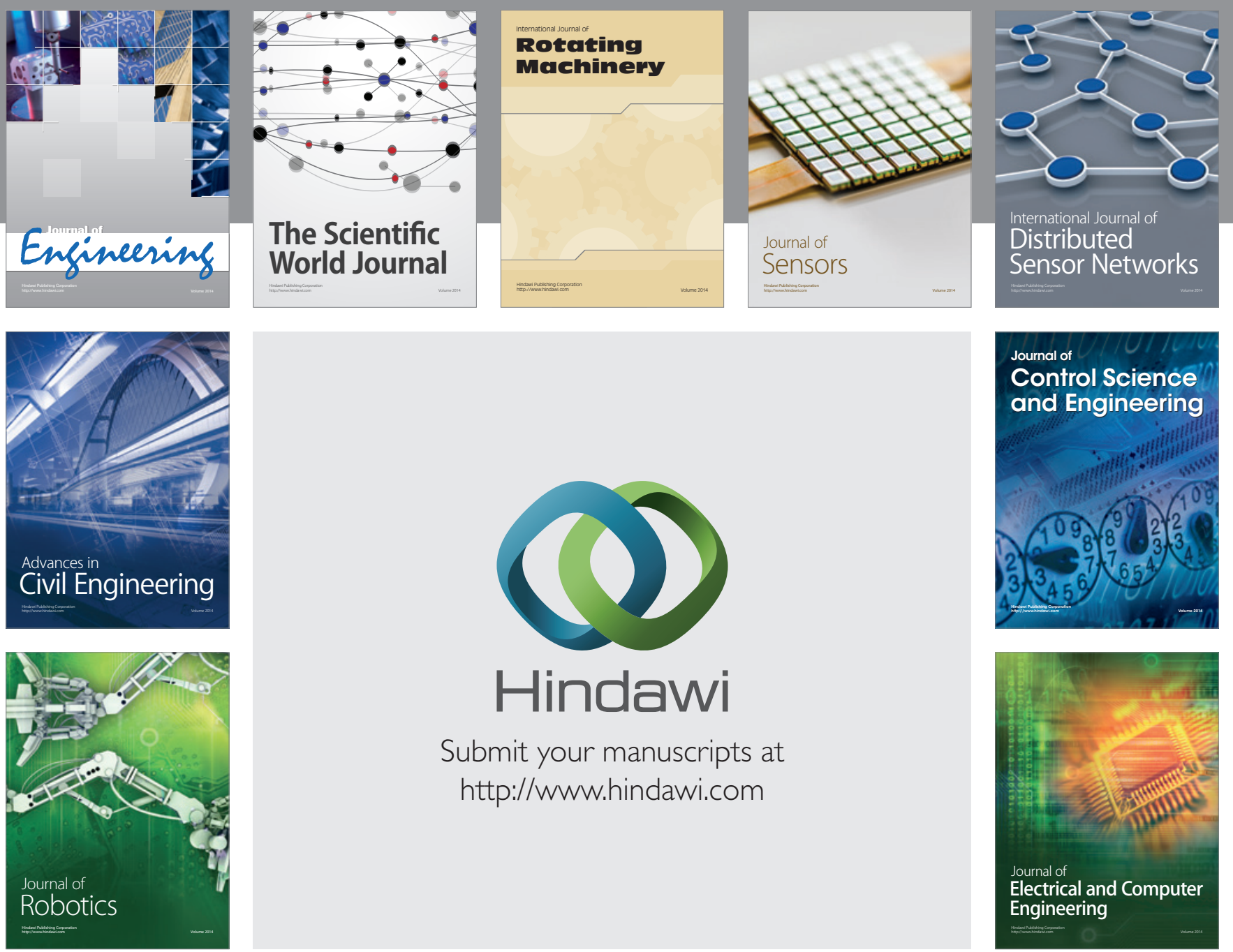

Submit your manuscripts at

http://www.hindawi.com
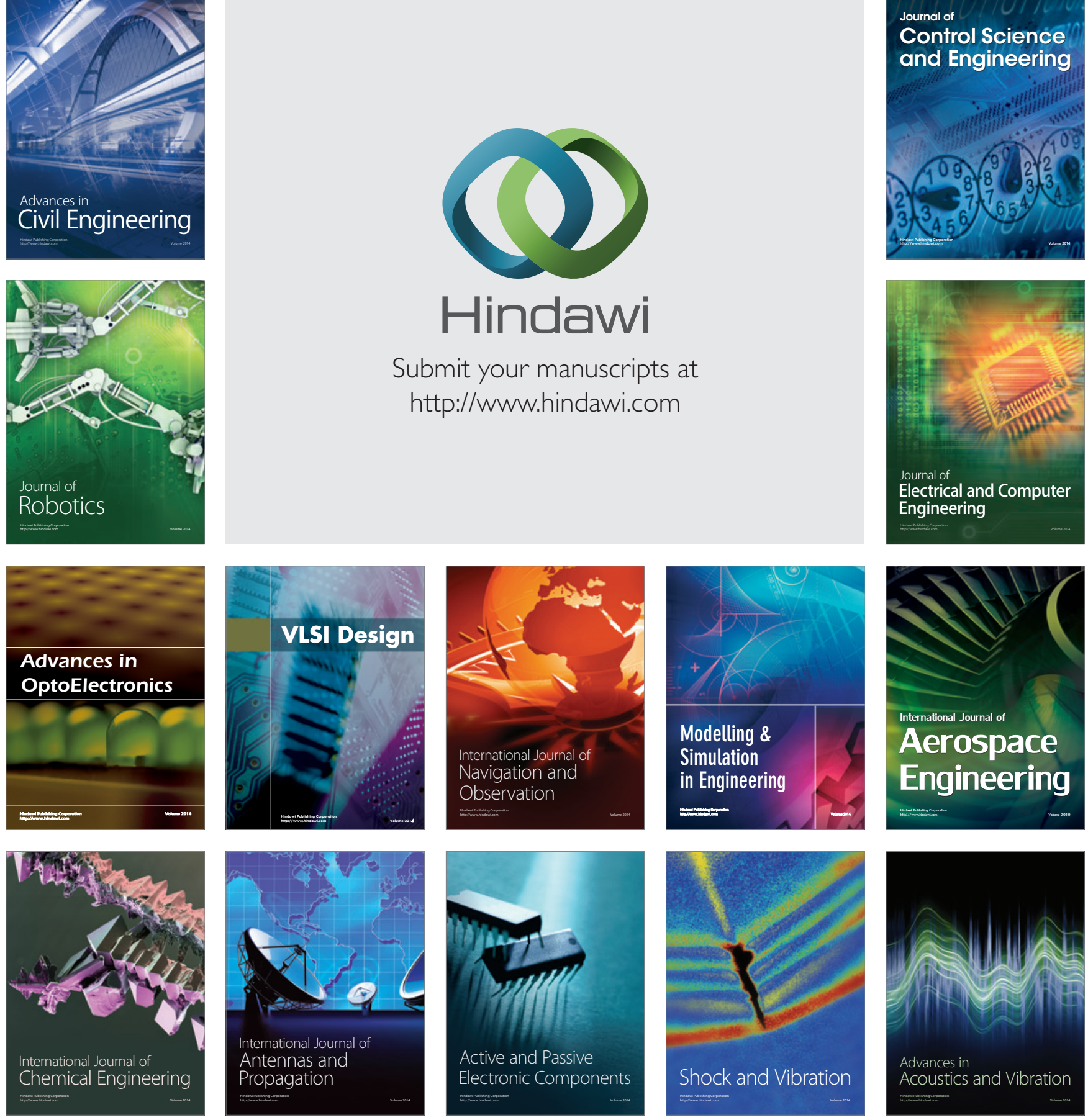